\title{
Sturm, Tanja
}

\section{Inklusion: Kritik und Herausforderung des schulischen Leistungsprinzips}

Erziehungswissenschaft 26 (2015) 51, S. 25-32

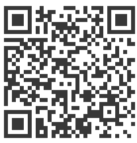

Quellenangabe/ Reference:

Sturm, Tanja: Inklusion: Kritik und Herausforderung des schulischen Leistungsprinzips - In:

Erziehungswissenschaft 26 (2015) 51, S. 25-32 - URN: urn:nbn:de:0111-pedocs-115680 - DOI: 10.25656/01:11568

https://nbn-resolving.org/urn:nbn:de:0111-pedocs-115680

https://doi.org/10.25656/01:11568

in Kooperation mit / in cooperation with:

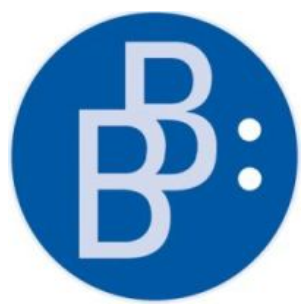

https://www.budrich.de

\section{Nutzungsbedingungen}

Gewährt wird ein nicht exklusives, nicht übertragbares, persönliches und beschränktes Recht auf Nutzung dieses Dokuments. Dieses Dokument ist ausschließlich für den persönlichen, nicht-kommerziellen Gebrauch bestimmt. Die Nutzung stellt keine Übertragung des Eigentumsrechts an diesem Dokument dar und gilt vorbehaltlich der folgenden Einschränkungen: Auf sämtlichen Kopien dieses Dokuments müssen alle Urheberrechtshinweise und sonstigen Hinweise auf gesetzlichen Schutz beibehalten werden. Sie dürfen dieses Dokument nicht in irgendeiner Weise abändern, noch dürfen Sie dieses Dokument für öffentliche oder kommerzielle Zwecke vervielfältigen, öffentlich ausstellen, aufführen, vertreiben oder anderweitig nutzen.

Mit der Verwendung dieses Dokuments erkennen Sie die Nutzungsbedingungen an.

\section{Terms of use}

We grant a non-exclusive, non-transferable, individual and limited right to using this document.

This document is solely intended for your personal, non-commercial use. Use of this document does not include any transfer of property rights and it is conditional to the following limitations: All of the copies of this documents must retain all copyright information and other information regarding legal protection. You are not allowed to alter this document in any way, to copy it for public or commercial purposes, to exhibit the document in public, to perform, distribute or otherwise use the document in public.

By using this particular document, you accept the above-stated conditions of use.

\section{Kontakt / Contact:}

\section{peDOCS}

DIPF | Leibniz-Institut für Bildungsforschung und Bildungsinformation Informationszentrum (IZ) Bildung

E-Mail: pedocs@dipf.de

Internet: www.pedocs.de

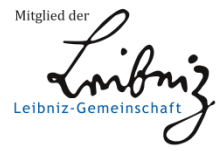




\section{INHALTSVERZEICHNIS}

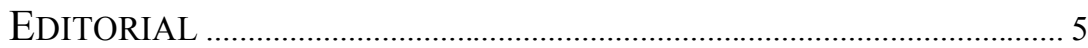

BEITRÄGE ZUM THEMA „INKLUSION - PERSPEKTIVE, HERAUSFORDERUNG UND PROBLEMATISIERUNG AUS SICHT DER ERZIEHUNGSWISSENSCHAFT“

Christian Lindmeier \& Birgit Lütje-Klose

Inklusion als Querschnittsaufgabe in der Erziehungswissenschaft.

Jessica M. Löser \& Rolf Werning

Inklusion - allgegenwärtig, kontrovers, diffus?

Tanja Sturm

Inklusion: Kritik und Herausforderung des schulischen

Leistungsprinzips .25

Jürgen Budde \& Merle Hummrich

Inklusion aus erziehungswissenschaftlicher Perspektive

Christian Lindmeier \& Bettina Lindmeier

Inklusion aus der Perspektive des rechtlichen und ethischen

Begründungsdiskurses

Safiye Yıld $ı$

Inklusion!? Was ist daran wahr?

Conny Melzer, Clemens Hillenbrand, David Sprenger

\& Thomas Hennemann

Aufgaben von Lehrkräften in inklusiven Bildungssystemen - Review

internationaler Studien

Thomas Häcker \& Maik Walm

Inklusion als Herausforderung an eine reflexive

Erziehungswissenschaft

Irene Moser \& Robert Schneider

Diskussionsbeitrag zur Debatte der DGfE „Inklusion als

Herausforderung für die Erziehungswissenschaft" .... 


\section{MitTEILUNGEN DES VORSTANDS}

Die Neuwahl des Fachkollegiums 109 „Erziehungswissenschaft“ bei der DFG - Zeit für eine Bilanz ....................................................................... 93

European Educational Research Association (EERA) ................................. 103

Weltbildungssemantik in the making? Die World Education Research Association (WERA).....

\section{BERICHTE AUS DEN SEKTIONEN}

Sektion 1 - Historische Bildungsforschung ................................................... 115

Sektion 2 - Allgemeine Erziehungswissenschaft ....................................... 122

Sektion 5 - Schulpädagogik .......................................................................... 123

Sektion 7 - Berufs- und Wirtschaftspädagogik ........................................... 127

Sektion 8-Sozialpädagogik und Pädagogik der frühen Kindheit............... 133

Sektion 11 - Frauen- und Geschlechterforschung in der Erziehungswissenschaft ……………………………................. 138

Sektion 12 - Medienpädagogik ................................................................ 140

NotIZEN

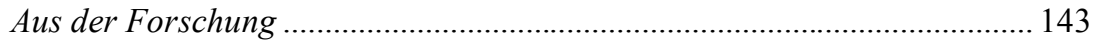

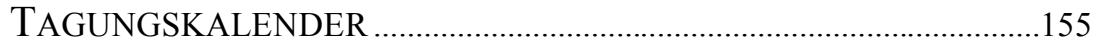

PERSONALIA

Nachruf auf Prof. em. Dr. Karlheinz Ingenkamp ....................................... 157 


\section{Inklusion: Kritik und Herausforderung des schulischen Leistungsprinzips}

Tanja Sturm

\section{Inklusion in Schule und Unterricht}

Inklusion ist zu einem viel diskutierten Begriff innerhalb der Erziehungswissenschaft avanciert. Anders als noch zu Beginn des Jahrtausends wird das Thema nicht mehr nur von einem Teil der Disziplin verhandelt (Tervooren 2001), sondern von unterschiedlichen erziehungswissenschaftlichen Diskurssträngen aufgegriffen, v.a. dem der Schulpädagogik (z.B. Cramer/Harant 2014). Gleichzeitig wird Kritik an den Möglichkeiten der Umsetzung schulischer Inklusion formuliert - nicht zuletzt aus der schulischen Praxis selbst. Auch die Auseinandersetzung der DGfE, die sich mit einer Fachtagung und einem Schwerpunktheft dem Thema widmet, kann als Ausdruck dieses Bedeutungszuwachses sowie - das geht aus der Tagungsankündigung hervor offener Fragen gewertet werden.

Inklusion wird vielfach in Bezug auf die gemeinsame Beschulung von Kindern und Jugendlichen mit und ohne „sonderpädagogischen Förderbedarf" verstanden, wie sie bildungspolitisch und/oder mit Bezug auf die UNBRK (UN 2006, 2008) gedeutet wird (siehe Häcker/Walm in diesem Heft). Dieser Beitrag legt den Schwerpunkt auf den erziehungswissenschaftlichen Diskurs schulischer Inklusion, der in „,theoretischer Kontinuität“ (Lütje-Klose/Urban 2014, S. 114) zu dem der Integration steht und Strukturen und Praktiken der schulischen und unterrichtlichen Hervorbringung und Bearbeitung von Behinderung und Benachteiligungen in den Blick nimmt. Dieser seit gut vierzig Jahren intensiv geführte Diskurs zeigt die Notwendigkeit zur kritischen Auseinandersetzung mit dem Schule und Unterricht dominierenden Konzept individueller, schulischer Leistung auf. Ziel dieses Beitrags ist es, beide Diskurse - zu Inklusion und zu schulischer Leistung - kurz vorzustellen und daran anschließend Herausforderungen und Kritik, die sich der Erziehungswissenschaft als Forschungs- und Ausbildungsdisziplin im Kontext eines an Leistung orientierten Schulsystems stellen, zu skizzieren.

\section{Inklusion und Inklusionspädagogik}

Die erziehungswissenschaftliche Auseinandersetzung mit Inklusion bzw. Integration in Schule und Unterricht, die seit mehr als 100 Jahre geführt wird (Ellger-Rüttgardt 2008, S. 108), weist zwei zentrale Bezüge auf: das Behin- 
derungsverständnis und die kritische Auseinandersetzung des schulisch-organisatorischen Umgangs mit Behinderungen (Sturm 2015b).

Seit den 1970er Jahren hat Integration in der Erziehungswissenschaft an Relevanz gewonnen; Ausgangspunkt hierfür waren häufig von Elterninitiativen initiierte „Schulversuche“ einiger westdeutscher Bundesländer, die die gemeinsame Beschulung von Schülerinnen und Schüler mit und ohne „Sonderschulbedarf" vorsahen. Diese wurden wissenschaftlich begleitet und es wurden didaktische Integrationskonzepte formuliert (z.B. Feuser 1999; Heyer et al. 1990; Schuck et al. 1998). „Sonderschulbedarf" verweist auf ein Behinderungsverständnis, das Behinderungen Personen zuschreibt und zugleich Bezug auf die Strukturen des Schulsystems als Förder- respektive Bildungsort nimmt. Etwa zur gleichen Zeit setzte sich im erziehungswissenschaftlichen Diskurs zu Behinderung ein Verständnis durch, das eine Abkehr von einer essentialistischen Perspektive zugunsten eines sozialwissenschaftlichen Verständnisses darstellt. Letztgenanntes verortet Behinderungen nicht in Personen, sondern verortet sie in Interaktionen: Behinderungen können situativ oder kontinuierlich auftreten (Tervooren 2000, S. 317). Überdauernde Formen, die wiederholt zu Ausschluss - häufig der gleichen Personen(gruppen) - führen, können sich zu Erfahrungen der Personen verdichten, die wiederum weiteren Handlungen zugrunde liegen. Behinderung wird dabei in Relation zu kontextuellen Erwartungen, wie z.B. jener, spezifische Leistung in der Schule zu erbringen, verstanden. Vor diesem Hintergrund formierte sich, z.T. in dezidierter Abgrenzung gegenüber der Sonderpädagogik, die Integrationspädagogik (z.B. Eberwein 1998). Ebenso wie materialistische Perspektiven der Behindertenpädagogik (z.B. Feuser 1995) plädierte sie für die Veränderung der Allgemeinen Pädagogik zugunsten einer Verantwortung für alle Schülerinnen und Schüler sowie für die Überwindung der disziplinären Trennung von Allgemeiner und Sonderpädagogik (Eberwein 1988).

Das menschenrechtliche Behinderungsverständnis der UN-BRK distanziert sich von einer essentialistischen Perspektive und konzipiert Defizite interaktiv, als ausgrenzenden und diskriminierenden Umgang mit Differenzen (Bielefeldt 2009, S. 6f.). Vor dieser Folie des Behinderungsverständnisses wird Inklusion an dem Ziel orientiert, situative und überdauernde Formen von Behinderung zu überwinden (Ainscow 2008). Im deutschsprachigen Diskurs hat sich Inklusion als Begriff gegenüber Integration durchgesetzt; dieser Wandel gründet in der Bezugnahme auf supranationale Dokumente wie UNBRK und Salamanca-Erklärung (UNESCO 1994) einerseits und auf den englischsprachigen Fachdiskurs zu Inclusive Education (Allan/Slee 2008) andererseits. Die aufgezeigten Verständnisse von Behinderung und Inklusion eröffnen die Möglichkeit, Behinderungen in Schule und Unterricht über die schuladministrative Differenzdimension des ,sonderpädagogischen Förderbedarfs" hinaus zu betrachten, also v.a. behinderte Lern- und Bildungsprozesse. Inklusion - in Relation zu Exklusion - kann somit analytisch herange- 
zogen werden, um unterrichtliche Interaktionen und/oder schulische Strukturen hinsichtlich behindernder Strukturen und Praktiken zu betrachten.

\section{Die leistungsorientierte Schule}

Die bildungspolitische (Heraus-)Forderung der Gestaltung einer an Inklusion orientierten Schule trifft auf die sog. Regelschule, die ihrerseits, auch ohne einen Inklusionsanspruch, soziale Ungleichheit und Benachteiligungen (re)produziert - meist ohne dass ihre Strukturen und Praktiken grundlegend hinterfragt würden (Becker 2015, S. 126ff.). Das die soziale und Bildungsungleichheit legitimierende Konstrukt schulischer Leistung wird v.a. individuell zugeschrieben (Stojanov 2015). Die zentrale Bedeutung, die Leistung(sunterschieden) im deutschen Schulsystem zukommt, die sich sowohl schulorganisatorisch und strukturell als auch in einer unterrichtlichen Eigendynamik findet, ist in anderen Ländern wie z.B. den angelsächsischen und skandinavischen nicht in vergleichbarer Weise konstitutiv (Biermann/Powell 2014; Stojanov 2015).

Leistung ist nicht nur ein zentraler schulischer, sondern auch ein gesellschaftlicher Bezugspunkt. Die Leistungsgesellschaft basiert auf der Idee, dass die (ungleiche) Verteilung von Macht und Gütern nach individuell erbrachter Leistung erfolgt und somit ein ,egalitäres gesellschaftliches Inklusionsprinzip“ (Schäfer/Thompson 2015, S. 8) darstellt, das auf der Annahme beruht, dass höhere Leistung zu entsprechend höheren gesellschaftlichen Positionen und/oder ökonomischen Möglichkeiten führt. Dieses Prinzip findet sich auch in der Schule: Höhere Leistung verspricht den Zugang zu exklusive(re)n Bildungsgängen und der in ihnen vergebenen Bildungszertifikaten, die ihrerseits Möglichkeiten eröffnen (ebd.).

Dieses Leistungsverständnis und das mit ihm verbundene emanzipatorischere Potenzial sind brüchig, da empirisch belegt ist, dass schulischer Bildungs(miss)erfolg nicht allein auf indiviudelle Leistung zurückzuführen ist, sondern in Korrelation zu gesellschaftlich relevanten Differenz- und Ungleichheitsdimensionen (Baumert et al. 2001) und schulischen Kontextfaktoren steht. Die Schule - und damit auch der Unterricht - beteiligen sich an der Reproduktion sozialer Ungleichheit in der Gesellschaft (Bourdieu 2001).

Das Leistungsprinzip findet sich schulorganisatorisch sowohl in der Jahrgangsklasse als auch in den unterschiedlichen, nach Leistungserwartungen differenzierten Bildungsgängen. Beide ermöglichen und erfordern zugleich, die Schülerinnen und Schüler kontinuierlich miteinander sowie gegenüber normierten, zeitlich formulierten Erwartungen $\mathrm{zu}$ vergleichen (Reh et al. 2015). Dabei wird nicht nur die Verantwortung der Lehrpersonen an der $\mathrm{Zu}-$ schreibung von Leistung ausgeblendet (Breidenstein 2011), sondern Leistung 
vollzieht sich in der Schule als Wettbewerb um bessere Positionen (Schäfer/ Thompson 2015). In der Schule wird Leistung hierarchisch konzipiert, indem die Schülerinnen und Schüler anhand von Leistungsmaßstäben geordnet werden, und so der gesellschaftliche Raum strukturiert wird. Dies verstärkt sich aktuell durch Formen der schulischen Leistungsmessung, die - unter Ausblendung des gesellschaftlichen, unterrichtlichen sowie des biographisch-sozialen Kontextes - „objektiv“ Leistung messen. Zugleich implizieren diese Entwicklungen, dass Bildung auf fachbezogene Kompetenzen reduziert wird (Angermuller/Maeße 2015, S. 63ff.).

Auf der Mikroebene unterrichtlicher Interaktionen zeigt sich, dass Leistung den „Kern pädagogischer Ordnungen“ (Rabenstein et al. 2013, S. 674) darstellt und die permanente Leistungsbewertung eine Eigenlogik entwickelt hat, die im Zusammenhang mit dem strukturellen Problem steht, dass Noten legitimiert und als "gerecht“ anerkannt werden (müssen) (Breidenstein 2011). Lehrpersonen und Schülerinnen und Schüler verfügen über ein handlungspraktisches Leistungsverständnis, das diese individuell zuschreibt und - auch in dem Anspruch nach inklusiven Klassen - hierarchische Unterscheidungen hervorbringt. Die unterrichtliche Bearbeitung von als „defizitär“ wahrgenommenen Differenzen orientiert sich am Ziel der Kompensation gegenüber einer (klassenbezogenen) Norm (z.B. Sturm/Wagner-Willi 2015). Behindert werden dabei die Lern- und Bildungsprozesse jener Schülerinnen und Schüler, die die Norm bereits erreicht haben und/oder die derjenigen, die die Erwartungen (noch) nicht erfüllen, indem ihnen keine Lehrangebote unterbreitet werden (Sturm 2012). Beide Bearbeitungsformen sind an einem Abbau der Differenzen orientiert. So machen die Schülerinnen und Schüler die Erfahrungen, dass (Leistungs-)Differenzen unterrichtlich nicht erwünscht. Die Verbindung beider Aspekte birgt das Potenzial, spezifische Schülerinnen(gruppen) und Schüler(gruppen) aufgrund ihres schulischen Misserfolgs zu marginalisieren (Sturm 2015a).

Das Leistungsprinzip, das sich schulorganisatorisch durchgesetzt hat, wirkt zugleich subjektivierend, indem von den Schülerinnen und Schülern kontinuierlich die Bereitschaft zur Leistungserbringung gefordert wird (Reh et al. 2015). Vor dem Hintergrund aktueller gesellschaftlich-ökonomischer Rahmenbedingungen und Entwicklungen erfährt schulische Leistung eine Bedeutungszunahme (Angermuller/Maeße 2015) und damit verbunden auch die individuelle Zuschreibung und Prekarität, die den Einzelnen bei nicht erbrachter Leistung droht. 


\section{Erziehungswissenschaftliche Herausforderungen in Forschung und Lehre}

Vor dem Hintergrund der skizzierten Verständnisse von Inklusion und Leistung im Kontext von Schule wird deutlich, dass sie in vielerlei Hinsicht konträr zu einander liegen bzw. auf unterschiedliche Aspekte schulischer Realität, gesellschaftlicher Herausforderungen und Entwicklungen verweisen. Mithin stellt der Anspruch auf Inklusion eine Kritik am aktuell praktizierten und realisierten Leistungsverständnis der Schule dar. Vor diesem Hintergrund sehe ich die Herausforderung der Erziehungswissenschaft v.a. in der Reflexion beider Konstituenten und der Folgen der gleichzeitigen Umsetzung beider, wie z.B. Kritik an der Hierarchisierung von Leistungsdifferenzen durch den Anspruch auf Inklusion, da die Hierarchisierung insofern behindernd wirkt, als sie Lernende mit unterschiedlichen biographischen Erfahrungen in ihrer lernenden Auseinandersetzung mit sozialen und materialen Gegenständen als „besser“ und „schlechter“" charakterisiert.

Praxeologisch fundierte Theorien, wie die Praxeologische Wissenssoziologie (Bohnsack 2010) eröffnen durch die Unterscheidung von sprachlich-reflexiv zugänglichem und handlungspraktischem, in der Regel nicht reflexivzugänglichem Wissen eine differenzierte Analyse schulischer und unterrichtlicher Strukturen und Praktiken. In Bezug auf Inklusion und Leistung eröffnen sie die Möglichkeit, zwischen dem formalen Anspruch auf Inklusion einerseits und der handlungspraktischen Umsetzung - im Kontext einer an Leistung orientierten Schule - andererseits zu unterscheiden und so Behinderungen von Lern- und Bildungsprozessen zu beschreiben (Sturm/WagnerWilli 2015).

Die Erziehungswissenschaft und v.a. die Schulpädagogik, die nicht allein gesellschaftliche Veränderungen - wie den Abbau sozialer Ungleichheit wird realisieren können, sehe ich vor dem Hintergrund der Ausführungen herausgefordert, sich reflexiv des gesellschaftlichen und schulischen Rahmens zu vergewissern und zu positionieren, innerhalb derer Lern- und Bildungsprozesse organisatorisch eingebettet und pädagogisch unterstützt bzw. initiiert anstatt behindert werden.

Vor dieser Folie wäre zu überlegen, wie der Anspruch eines reflektierten und egalitären Miteinanders von Differenz und Gemeinsamkeit in Schule und Unterricht aussehen kann (siehe Häcker \& Walm in diesem Band). Als rahmendes Konzept beschreibt Inklusion in diesem Sinne eine Sichtweise und praktische Haltung und keinen Zustand, der, einmal erreicht, als vorhanden gelten kann.

Konkrete Ansatzpunkte für die erziehungswissenschaftliche Forschung und Lehre liegen in der Breite der Erziehungswissenschaft und der Schulpädagogik. Hieran anknüpfend sehe ich die Potenziale eines erziehungswissenschaftlichen Beitrags zu einer Gesellschaft bzw. dem gesellschaftlichen Teil- 
bereich des Schulsystems, in denen Differenzen ,,ausdrücklich bejaht und darüber hinaus als Quelle möglicher kultureller Bereicherung wertgeschätzt“ werden (Bielefeldt 2009, S. 6f.), und die eine Basis für ein demokratisches Miteinander darstellen.

Tanja Sturm, Prof. Dr., ist Hochschullehrerin für Inklusive Didaktik und Heterogenität an der Fachhochschule Nordwestschweiz.

\section{Literatur}

Ainscow, Mel (2008): Teaching for diversity. The next big challenge. In: Connelly, F. M./Fang He, M./Phillion, J. (Hrsg.): The Sage Handbook of Curriculum and Instruction. Los Angeles u.a.: SAGE, S. 240-258.

Allan, Julie/Slee, Roger (2008): Doing Inclusive Education Research. Rotterdam: Sense Publishers.

Angermuller, Johannes/Maeße, Jens (2015): Regieren durch Leistung. Zur Verschleierung des Sozialen in der Numerokratie. In: Schäfer, A./ Thompson, C. (Hrsg.): Leistung. Paderborn: Ferdinand Schöningh, S. 61108.

Baumert, Jürgen/Klieme, Eckhard/Neubrand, Michael/Prenzel, Manfred/ Schiefele, Ulrich/Schneider, Wolfgang/Stanat, Petra/Tillmann, KlausJürgen/Weiß, Manfred (2001): PISA 2000. Basiskompetenzen von Schülerinnen und Schülern im internationalen Vergleich. Opladen: Leske + Budrich.

Becker, Uwe (2015): Die Inklusionslüge. Behinderung im flexiblen Kapitalismus. Bielefeld: transcript.

Bielefeldt, Heiner (2009): Zum Innovationspotenzial der UN-Behindertenrechtskonvention. http://www.institut-fuer-menschenrechte.de/uploads/tx _commerce/essay_no_5_zum_innovationspotenzial_der_un_behinderten rechtskonvention_auf13.pdf [Z̄ugriff: 09. August 2015].

Biermann, Julia/Powell, Justin J. W. (2014): Institutionelle Dimensionen inklusiver Schulbildung - Herausforderungen der UN-Behindertenrechtskonvention für Deutschland, Island und Schweden im Vergleich. In: Zeitschrift für Erziehungswissenschaft 17, 4, S. 679-700.

Bohnsack, Ralf (2010): Rekonstruktive Sozialforschung. Einführung in qualitative Methoden. Opladen, Farmington Hills: Verlag Barbara Budrich.

Bourdieu, Pierre (2001): Wie die Kultur zum Bauern kommt. Über Bildung, Schule und Politik. Hamburg: VSA-Verlag.

Breidenstein, Georg (2011): Zusammenfassende und vergleichende Betrachtungen. In: Zaborowski, K. U./Meier, M./Breidenstein, G. (Hrsg.): Leistungsbewertung und Unterricht. Ethnographische Studien zur Bewer- 
tungspraxis in Gymnasien und Sekundarschule. Wiesbaden: VS Verlag für Sozialwissenschaften. S. 345-366.

Cramer, Colin/Harant, Martin (2014): Inklusion - Interdisziplinäre Kritik und Perspektiven von Begriff und Gegenstand. In: Zeitschrift für Erziehungswissenschaft 17, 4, S. 639-659.

Eberwein, Hans (1988): Vorbemerkungen. In: Eberwein, H. (Hrsg.): Behinderte und Nichtbehinderte lernen gemeinsam. Handbuch der Integrationspädagogik. Weinheim, Basel: Beltz, S. 9-10.

Eberwein, Hans (1998): Sonder- und Rehabilitationspädagogik - eine Pädagogik für „Behinderte“ oder gegen Behinderungen? Sind Sonderschulen verfassungswidrig? In: Eberwein, H./Sasse, A. (Hrsg.): Behindert sein oder behindert werden? Interdisziplinäre Analysen zum Behinderungsbegriff. Neuwied, Kriftel, Berlin: Luchterhand, S. 66-95.

Ellger-Rüttgardt, Sieglind L. (2008): Geschichte der Sonderpädagogik. Eine Einführung. München: Ernst Reinhardt.

Feuser, Georg (1995): Behinderte Kinder und Jugendliche. Zwischen Integration und Aussonderung. Darmstadt: Wissenschaftliche Buchgesellschaft.

Feuser, Georag (1999): Modelle der Integration: Fortschritt oder Inflationierung des Integrationsanliegens? In: Flieger, P./Lener, G./Leskovat, E./Jäger, M./Zuber, J. (Hrsg.): Behinderung. Integration in der Schule. Wien: Verein der Förderer der Schulhefte, S. 29-42.

Heyer, Peter/Preuss-Lausitz, Ulf/Zielke, Gisela (1990): Wohnortnahe Integration. Gemeinsame Erziehung behinderter und nichtbehinderter Kinder in der Uckermark-Grundschule in Berlin. Weinheim, München: Juventa.

Lütje-Klose, Birgit/Urban, Melanie (2014): Professionelle Kooperation als wesentliche Bedingung inklusiver Schul- und Unterrichtsentwicklung. Teil 1: Grundlagen und Modelle inklusiver Kooperation. In: Vierteljahresschrift für Heilpädagogik und ihre Nachbargebiete 83, 2, S. 112-123.

Rabenstein, Kerstin/Reh, Sabine/Ricken, Norbert/Idel, Till-Sebastian (2013): Ethnographie pädagogischer Differenzordnungen. Methodologische Probleme einer ethnographischen Erforschung der sozial selektiven Herstellung von Schulerfolg im Unterricht. In: Zeitschrift für Pädagogik 59, 5, S. 668-690.

Reh, Sabine/Berdelmann, Kathrin/Scholz, Joachim (2015): Der Ehrtrieb und unterrichtliche Honorierungspraktiken im Schulwesen um 1800. Die Entstehung des Leistungs-Dispositivs. In: Schäfer, A./Thompson, C. (Hrsg.): Leistung. Paderborn: Ferdinand Schöningh, S. 37-60.

Schäfer, Alfred/Thompson, Christiane (2015): Leistung - eine Einleitung. In: Schäfer, A./Thompson, C. (Hrsg.): Leistung. Paderborn: Ferdinand Schöningh, S. 7-35.

Schuck, Karl Dieter/Rauer, Wulf/Hinz, Andreas/Katzenbach, Dieter/Wocken, Hans/Wudtke, Hubert (1998): Die Integrative Grundschule im sozialen 
Brennpunkt. Ergebnisse eines Hamburger Schulversuchs. Hamburg: Hamburger Buchwerkstatt.

Stojanov, Krassimir M. (2015): Leistung - ein irreführender Begriff im Diskurs über Bildungsgerechtigkeit. In: Schäfer, A./Thompson, C. (Hrsg.): Leistung. Paderborn: Ferdinand Schöningh, S. 135-150.

Sturm, Tanja (2012): Praxeologische Unterrichtsforschung und ihr Beitrag zu inklusivem Unterricht. In: Inklusion online, (1-2). http://www.inklusiononline.net/index.php/inklusion/article/view/151/143 [Zugriff: 09. August 2015].

Sturm, Tanja (2015a): Herstellung und Bearbeitung von Differenz im inklusiven Unterricht. In: Bräu, K./Schlickum, C. (Hrsg.): Soziale Konstruktionen im Kontext von Schule und Unterricht. Opladen, Farmington Hills: Verlag Barbara Budrich, S. 213-224.

Sturm, Tanja (2015b): Von der Sonderpädagogik zur Inklusiven Pädagogik Phasen der Entwicklung Inklusiver Bildung. In: Hedderich, I./Biewer, G. /Hollenweger, J./Markowetz, R. (Hrsg.): Handbuch Inklusion und Sonderpädagogik. Bad Heilbrunn: Klinkhardt. Im Erscheinen.

Sturm, Tanja/Wagner-Willi, Monika (2015): „Leistungsdifferenzen“ im Unterrichtsmilieu einer inklusiven Schule der Sekundarstufe I in der Schweiz. In: Zeitschrift für Qualitative Forschung, 16. Im Erscheinen.

Tervooren, Anja (2000): Differenz anders gesehen: Studien zu Behinderung. In: Viertelsjahresschrift für Heilpädagogik und ihre Nachbargebiete 69, 3, S. 316-319.

Tervooren, Anja (2001): Pädagogik der Differenz oder differenzierte Pädagogik? Die Kategorie Behinderung als integraler Bestandteil von Bildung. In: Tervooren, A./Hartmann, J./Fritzsche, B./Schmidt, A. (Hrsg.): Dekonstruktive Pädagogik. Erziehungswissenschaftiche Debatten unter poststrukturalistischen Perspektiven. Wiesbaden: Springer Fachmedien, S. 201-216.

UN (2006/2008): Übereinkommen über die Rechte von Menschen mit Behinderungen. (dreisprachige Fassung im Bundesgesetzblatt Teil II Nr. 35 vom 31.12.2008). (Manuskriptdruck). http://www.bgbl.de/xaver/bgbl/sta rt.xav?startbk $=$ Bundesanzeiger BGBl\&bk=Bundesanzeiger BGBl\&start $=/ / * \% 5 \mathrm{~B} @$ attr_id=\%27bgbl208s $1419 . p d f \% 27 \% 5 \mathrm{D}$ - _ bgb $\bar{b} \mathrm{O} \% 2 \mathrm{~F} \% 2 \mathrm{~F}$ *\%5B\%40attr_id\%3D\%27bgbl208s1419.pdf\%27\%5D_143668081055 5 [Zugriff: 27. März 2010].

UNESCO (1994): The Salamanca Statement and Framework for Action on Special Needs Education. World Conference of Special Needs Education: Access and Quality. http://unesdoc.unesco.org/images/0009/000984 /098427eo.pdf [Zugriff: 14. März 2015]. 